\title{
La Reserva de la Biósfera Cinturón Andino, Colombia. ¿Una región modelo de estrategias de adaptación al cambio climático y el desarrollo regional sustentable? 1
}

\author{
Axel Borsdorf ${ }^{2}$, Martin Mergili ${ }^{3}$ y Luis Alfonso Ortega ${ }^{4}$
}

\begin{abstract}
RESUMEN
La cuenca del río Piedras en la Reserva de la Biósfera Cinturón Andino puede considerarse un laboratorio para el desarrollo de iniciativas orientadas a la aplicación de técnicas de agricultura sustentable, adaptadas a las condiciones del cambio climático. Las estrategias utilizadas se basan en el fortalecimiento del capital social y buscan mejorar las condiciones de vida de la población rural y conservar la biodiversidad y servicios ecosistémicos. Este trabajo identifica indicadores de cambio climático evidenciados por la población local y evalúa el éxito de las estrategias utilizadas, para considerarlas en otros contextos de áreas montañosas. Los resultados indican que las estrategias aplicadas son exitosas para asegurar la producción agrícola y los servicios ecosistémicos en un contexto de cambio climático. Sin embargo, mayores avances en la calidad de vida y seguridad social son todavía dificultados por las limitaciones financieras.
\end{abstract}

Palabras clave: Reservas de la biósfera, adaptación al cambio climático, servicios ecosistémicos, agricultura de montaña, organizaciones de la sociedad civil.

\begin{abstract}
The Río Las Piedras basin in the Biosphere Reserve Cinturón Andino (Colombia) serves as a laboratory for initiatives directed towards sustainable agricultural techniques adapted to climate change conditions. Building on an improved social capital, the strategies applied aim at the improvement of the livelihood of the rural population and at the conservation of biodiversity and other ecosystem services. The present paper identifies the indicators of climate change experienced by the local population and tries to evaluate the success of the strategies applied in order to assess the applicability of such strategies to other mountain areas. The results indicate that the strategies applied are largely successful in ensuring the agricultural production and the ecosystem services also under changing climatic conditions. However, further improvement of the livelihood and social security are impeded by limited financial resources.
\end{abstract}

Key words: Biosphere reserve, climate change adaptation, ecosystem services, mountain agriculture, civil society organizations.

\footnotetext{
1 Los autores agradecen a los estudiantes de Geografía de la Universidad de Innsbruck, quienes realizaron gran parte de las entrevistas y a los campesinos de la cuenca del río Piedras y sus familias por su amabilidad. Además, agradecen a Liliana Recaman de la Fundación Río Piedras-Acueducto de Popayán y Apolinar Figueroa de la Universidad del Cauca por su apoyo en la investigación, y Carla Marchant por su colaboración en la traducción de este trabajo. Artículo recibido el 29 de marzo de 2012, aceptado
}

el 17 de noviembre de 2012 y corregido el 31 de enero de 2013.

2 Institute for Interdisciplinary Mountain Research, Austrian Academy of Sciences; Institute of Geography, University of Innsbruck (Austria). E-mail: axel.borsdorf@uibk.ac.at

3 Institute of Applied Geology, BOKU University of Natural Resources and Life Sciences (Austria).

E-mail: martin.mergili@boku.ac.at

4 Fundación Ecohabitats (Colombia)

E-mail: luisortegafernandez@gmail.com 
La Reserva de la Biósfera Cinturón Andino, ubicada en la cordillera Central de Colombia, representa un área montañosa tropical de gran valor ecológico (Figueroa y Valencia 2009). La riqueza de esta zona se encuentra en riesgo, tanto por cambios medioambientales, particularmente desencadenados por el cambio climático, como por la aplicación de inadecuadas técnicas de manejo del suelo (Borsdorf et al., 2011). Asimismo, un desarrollo ecológico y socioeconómico sustentable en el área, fue dificultado por diversos conflictos armados, tanto entre las fuerzas guerrilleras de las FARC y paramilitares, como también entre grupos campesinos e indígenas durante décadas. Sin embargo, a principios del siglo XXI, la región experimenta un proceso de pacificación de los conflictos armados, lo cual permite a esta área convertirse en una región modelo para la cooperación entre grupos de campesinos e indígenas y a su vez para la promoción e implementación de técnicas de agricultura sustentable y estrategias de adaptación al cambio climático.

El presente trabajo tiene por objetivo (1) profundizar el conocimiento de los procesos de cambio medioambientales evidenciados por los campesinos; (2) aprender sobre estrategias de agricultura sustentable y adaptaciones al cambio climático aplicadas en el área, (3) evaluar el éxito de las estrategias aplicadas para mejorar la calidad de vida de la población y la conservación de los servicios ecosistémicos; y (4) valorar el potencial de las iniciativas aplicadas en la reserva de la biósfera Cinturón Andino, para ser transferidas a otras áreas montañosas. La investigación primaria fue desarrollada en la cuenca del río Piedras, cerca de la ciudad de Popayán, esto debido a que en este lugar existen las redes de contacto necesarias para llevar a cabo este tipo de estudio.

Existen escasas publicaciones que se ocupen de la cuenca del río Piedras. Figueroa et al. (2009) entregan una visión general de la biósfera y la biodiversidad de la zona, Borsdorf et al. (2011) publican una primera contribución sobre cambio climático y cooperación comunitaria. Otras reservas de la biósfera colombianas han sido también objeto de estudio como la Ciénaga Grande de Santa Marta, la cual fue analizada bajo los aspectos de la sustentabilidad por Vilardy y González (2011).
Las reservas de la biósfera fueron señaladas como regiones modelo para el desarrollo sustentable por Little et al. (1981); Batisse (1982, 1986, 1997), Schaaf (2003), Lange (2005), y Austrian MAB Committee (2011). Thiel \& Effler (2011) entregan un ejemplo ilustrativo de agricultura sustentable en la reserva de la biósfera Sierra Nevada de Santa Marta; Campbell et al. (2003) analizan las oportunidades de desarrollo participativo y conservación basada en el trabajo comunitario. Tomando en cuenta estos trabajos, el siguiente artículo responde la interrogante si el camino emprendido por las comunidades de campesinos e indígenas en la Reserva de la Biósfera Cinturón Andino es apropiado para asegurar la sustentabilidad y sus medios de vida. El caso de estudio seleccionado es un ejemplo exitoso que puede servir como modelo para otras regiones montañosas de Colombia.

\section{Reservas de la biósfera como un instrumento para el desarrollo rural sustentable}

Las reservas de la biósfera son el instrumento central del Programa Hombre y Biósfera de la Organización de las Naciones Unidas para la Educación, la Ciencia y la Cultura (UNESCO), el cual se instauró en 1976 para promover un desarrollo regional sustentable. Las reservas de la biósfera son áreas extensas y representativas de paisaje natural y cultural, las cuales deben ser resguardadas en el largo plazo. Ellas representan un reservorio de recursos genéticos y ecosistemas y son también áreas de uso sustentable del suelo, espacios de educación, investigación y recreación. Al año 2010, existen 564 reservas de la biósfera en 109 países.

Las reservas de la biósfera deben alcanzar tres objetivos fundamentales: conservación, desarrollo y logística (Schaaf, 2003). La función de conservación debe asegurar el rol del área como fuente genética y refugio de paisajes naturales y culturales; a su vez, se deben resguardar los servicios ecosistémicos (i.e. agua limpia). La función de desarrollo tiene por objetivo la aplicación de técnicas ecológicas y socioeconómicas sustentables en todos los sectores económicos. La función logística debe facilitar la investigación multidisciplinaria y las actividades educativas. La 
UNESCO ha definido lineamientos operacionales para las reservas de la biósfera como una manera de asegurar una mejor cooperación internacional. Esto también toma en cuenta leyes nacionales, las cuales juegan un importante rol en la situación actual de una reserva de la biósfera.

Para asegurar estas funciones, una reserva de la biósfera debe ser organizada en tres zonas (Figura $\mathrm{N}^{\circ} 1$ ): el núcleo central se caracteriza por permitir un mínimo de actividad antrópica, restringida solo a investigación y educación. Esta zona es designada para conservación de los ecosistemas naturales, los cuales no deben ser perturbados por las actividades permitidas. Asimismo, esta zona es protegida por la legislación del respectivo país, por ejemplo a través de la declaración de Parque Nacional. En la zona de amortiguación, el uso natural es permitido y actividades deseables son la agricultura orgánica o el ecoturismo. El modo de vida tradicional y las actividades económicas de la población, debe contribuir a la conservación del paisaje cultural de alto valor. La tercera zona, conocida como zona de desarrollo, es la más extensa y permite diferentes tipos de uso de suelo y asentamientos. En esta zona, un tra- bajo en conjunto entre la población, investigadores y la administración de la reserva de la biósfera es deseable, para asegurar un uso adecuado de los recursos naturales del área durante un periodo prolongado de tiempo.

\section{Escenario local}

La Reserva de la Biósfera Cinturón Andino abarca una superficie de $8550 \mathrm{~km}^{2}$, ocupando tanto, parte de la cordillera Central de Colombia, como del Macizo Colombiano, donde se reúnen la cordillera Central, Oriental y Occidental (Figura $\mathrm{N}^{\circ} 2$ a y Figura $\mathrm{N}^{\circ} 2 \mathrm{~b}$ ). Esta área extremadamente húmeda juega un rol esencial como reserva de agua y hot spot de biodiversidad (Borsdorf et al., 2011). La vegetación natural corresponde al bosque mesófilo de montaña, que se extiende hasta los 2.000 metros sobre el nivel del mar, bosque nuboso y las tierras altas del páramo andino (aproximadamente entre 3.200 a 4.000 metros sobre el nivel del mar; Figueroa et al., 2009). El páramo posee una particular capacidad de retención de agua, alimentando algunos de los ríos más largos del país, como por ejemplo el Magdalena, el Cauca y el Caquetá. Sin embargo, la intervención humana ha afectado la capacidad de almacenamiento

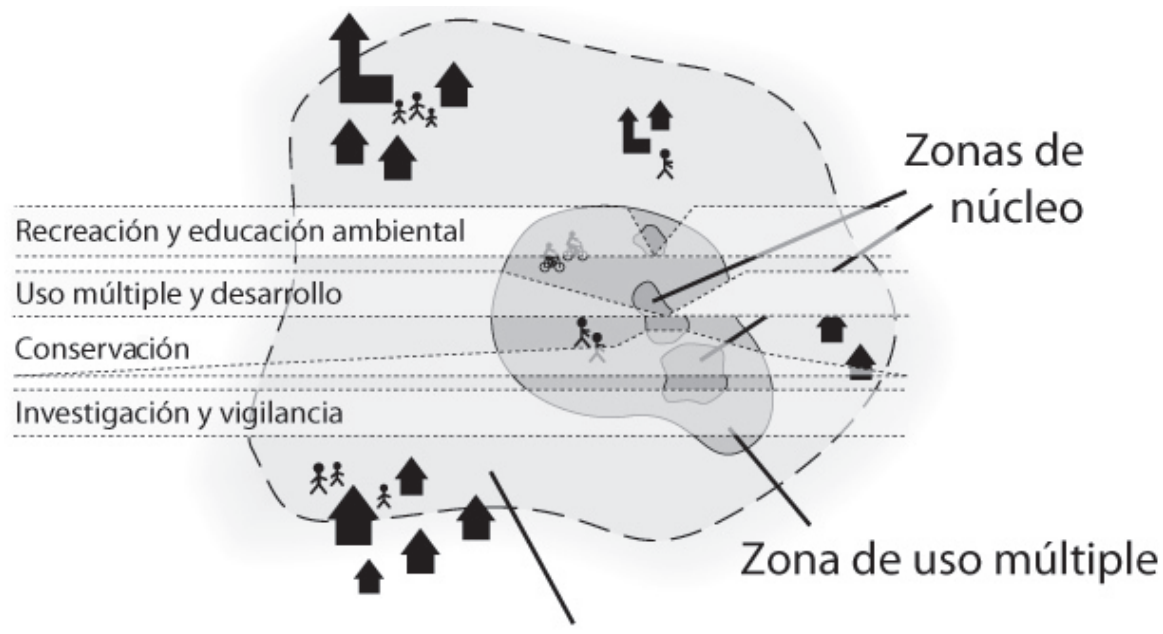

\section{Zona de transición}

Fuente: Elaboración propia en base a Lange (2005). 
Figura $\mathrm{N}^{\circ} 2$

Localización del área de estudio: (a) vista general, (b) Reserva de la Biósfera Cinturón Andino, (c) cuenca río Piedras. Los números 1 a 10 designan la localización de las fincas estudiadas
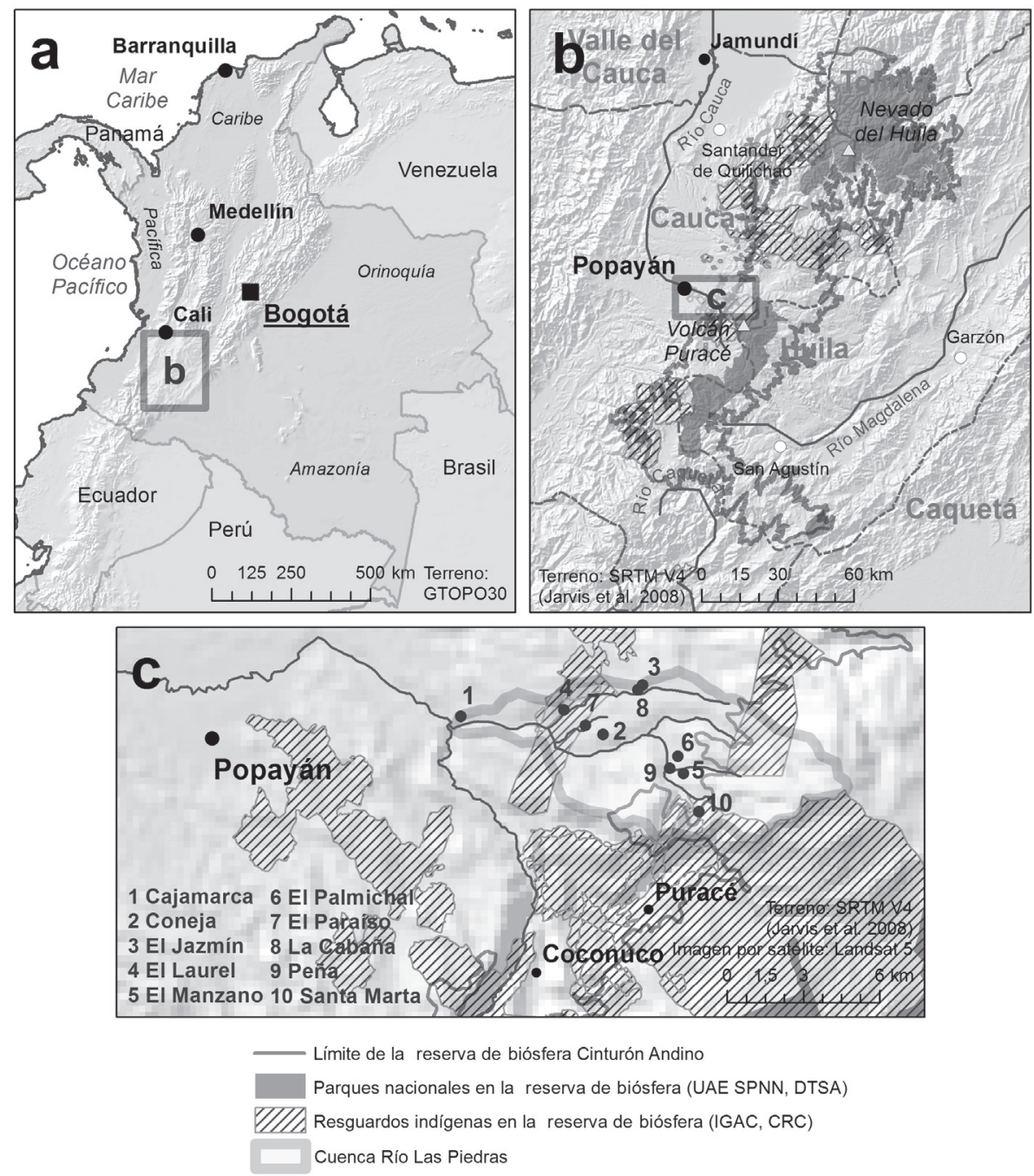

Fuente: Elaboración propia.

de agua de los ecosistemas y los procesos geomorfológicos que participan en la retención hídrica. Como consecuencia tanto la escorrentía, los patrones de transporte de sedimentos y la susceptibilidad a eventos de remoción en masa resultan alterados (Harden, 2006). Un número de estratovolcanes activos existe a lo largo de la cordillera Central, de los cuales, los más altos son el Nevado del Huila (5.364 m) y el Puracé $(4.756$ m).
El núcleo central de la reserva de la biósfera está conformado por tres parques nacionales (Nevado del Huila, Puracé y Cueva de los Guacharos), mientras que las áreas de amortiguación y de desarrollo han sido convertidas, en gran medida, a tierras de labranza. Parte de la población rural se declara a sí mismo como miembro de una comunidad indígena de la etnia Kokonuko (resguardos de Paletará, Purace, Kokonuko, Poblazón y 
Quintana); estas comunidades se organizan autónomamente, con su propia legislación y son denominados como indígenas, en contraste con los campesinos, que no pertenecen a este grupo (Borsdorf et al., 2011).

El presente artículo aborda la cuenca del río Piedras, ubicada en las zonas de amortiguación y de desarrollo de la Reserva de la Biósfera Cinturón Andino, en el lado occidental de la cordillera Central, cerca de la ciudad de Popayán (Figura $\mathrm{N}^{\circ} 2 \mathrm{c}$ ). La cuenca posee una dimensión de $58 \mathrm{~km}^{2}$ y se sitúa entre los $1.900 \mathrm{msnm}$ y $3.800 \mathrm{msnm}$ aproximadamente. Aunque recientemente se ha instalado una nueva estación en la cuenca del río Piedras, la estación meteorológica más cercana disponible y cuya información fue utilizada para caracterizar la temperatura y precipitación de la cuenca del río Piedras, se ubica en la ciudad de Popayán, con un promedio anual de precipitaciones de aprox. $2.000 \mathrm{~mm}$. Sin embargo, es esperable debido al efecto de la topografía y la altitud, que el monto de precipitaciones en la cuenca del río Piedras sea mayor (Figura $\mathrm{N}^{\circ} 3$ ).

Figura $\mathrm{N}^{\circ} 3$

Climograma de Popayán

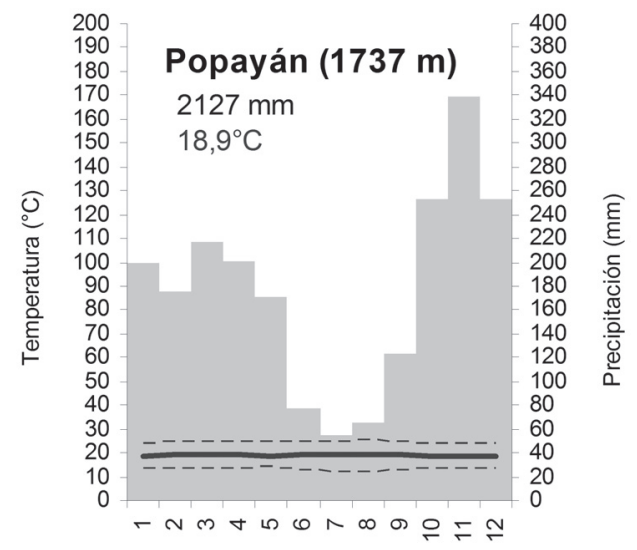

Fuente: Elaboración propia en base a datos del Instituto de Hidrología, Meteorología, y Estudios Ambientales (IDEAM).

La vegetación natural varía entre el bosque montano lluvioso, al bosque nuboso y el páramo. El bosque nuboso ocupa la mayor superficie. La mayor parte de los bosques han sido convertidos en tierra de labranza, algunas áreas remanentes son conservadas en el marco de la red de reservas. Sin embargo, muchos de estos ecosistemas protegidos corresponden a bosque secundario y relictos de roble (Quercus humboltii). Asimismo, una parte de la cuenca fue reforestada con especies arbóreas introducidas (Eucalyptus spp., Pinus radiata).

Hasta la década de 1990 e incluso en los primeros años del siglo XXI, la zona fue azotada parcialmente por un conflicto armado por la propiedad de la tierra, entre los indígenas y campesinos. La finca Santa Marta por ejemplo, fue adquirida legalmente por campesinos en 1992, pero tras la compra, fue sorpresivamente ocupada por indígenas durante años. En 1999, con el apoyo de la Fundación Río Piedras y la Dirección Territorial Surandina de la Unidad Administrativa de Parques Nacionales naturales se logró mitigar el conflicto, mediante la elaboración de un Pacto de Convivencia, que definió normas de coexistencia y respeto entre propiedades indígenas y campesinas y estableció las bases para procesos de pretensión y negociación de tierras privadas de terratenientes o del Estado. A principios del 2000 esta situación fue abordada y gestionada por organizaciones como la Asociación Campesina (ASOCAMPO).

\section{Iniciativas de agricultura sustentable en la cuenca del río Piedras}

El desarrollo de iniciativas locales para hacer frente a la adversa situación socioeconómica y política en la cuenca del río Piedras tiene una larga tradición. En el 2001 se fundó ASOCAMPO, la cual corresponde a una organización de la sociedad civil (Salamon et al., 2001). Con el advenimiento y establecimiento del neoliberalismo en Latinoamérica durante la década de 1980, los gobiernos nacionales delegaron muchas de sus funciones en el ámbito social y medioambiental. Este desarrollo privilegió la formación de organizaciones no gubernamentales sin fines de lucro u organizaciones de la sociedad civil (Salamon et al. 1999) muchas de las cuales se ocupan de asuntos medioambientales Bryant and Bailey (1997). Price et al. (1994) señalaron la existencia de aproximadamente 500 organizaciones medioambientales no gubernamentales en operación a nivel nacional 
y regional, así como otras miles a nivel local operando en Latinoamérica a principios de los noventa, comparadas con las aproximadamente 50 existentes a nivel nacional en 1979. En Colombia las asociaciones de usuarios de agua en el valle del Cauca son un ejemplo de este desarrollo. Este tipo de asociaciones se han creado en 12 cuencas tributarias, con el fin de proteger las fuentes de agua dulce. Estas operan como asociaciones públicoprivadas que recolectan fondos mientras que las obras son implementadas por la autoridad regional ambiental (Echavarría, 2002).

Inicialmente, el objetivo principal de ASOCAMPO era actuar como mediador en el conflicto entre indígenas y campesinos. Una década después, ASOCAMPO con el apoyo constante de la Fundación Río Piedras, se enfoca a la promoción de técnicas de agricultura sustentable bajo condiciones de cambio climático. Sus objetivos principales son: (1) conservar la integridad ecológica de las cuencas, (2) promover la agricultura ecológica, (3) impulsar la cooperación entre la comunidad y (4) conservar los recursos naturales en el área. En un sentido más amplio, las actividades incluyen también el mejoramiento de infraestructura y la capacitación de los campesinos. Al 2011, un total de 64 fincas se han unido a la asociación.

La Red de Reservas se encuentra estrechamente relacionada a las actividades de ASOCAMPO. Esta red es un instrumento perteneciente a las "Reservas Naturales de la Sociedad Civil" (RNSC), la única categoría de áreas protegidas en propiedad privada incluidas en las categoría del Sistema Nacional de Áreas Protegidas (SINAP) (Langholz \& Lassoie, 2000; Langholz et al. 2001). Las 64 fincas que conforman la Red de Reservas, deben destinar una parte de sus tierras agrícolas a la preservación del ecosistema. En la cuenca del río Piedras, es el bosque nuboso primario y secundario esencial para la conservación de la biodiversidad y para el abastecimiento de agua limpia. El tamaño de estas superficies protegidas varía entre $10^{-1}$ ha y $10^{1}$ ha.

\section{Metodología utilizada}

En primer lugar, se seleccionó una muestra de las fincas. Una finca es el nombre utilizado en Colombia para designar una propie- dad inmueble de carácter rural, la cual puede dedicarse a la producción agrícola o ganadera. La situación geográfica de las fincas es ilustrada en la Figura $N^{\circ} 2$, las características de las fincas son resumidas en el Cuadro $\mathrm{N}^{\circ}$ 2. Para ser seleccionada, la finca debía pertenecer tanto a ASOCAMPO como a la Red de Reservas y las familias debían estar preparadas para recibir a un equipo de entre 2 a 6 investigadores (dependiendo del tamaño de la finca y de su infraestructura), por un periodo de 3 días. La recopilación de información fue realizada, a través de observación directa y de entrevistas semiestructuradas. En cada una de las fincas seleccionadas, el equipo de investigadores se involucró, en la medida de lo posible, en las actividades y quehaceres cotidianos de la familia. Basándose de preguntas predefinidas, se buscó obtener información cualitativa y cuantitativa acerca del estado de la finca, la situación actual y las expectativas futuras (Cuadro $N^{\circ} 1$ ). Asimismo, se elaboraron cartas de uso del suelo en la finca y sobre la distribución de las dependencias del hogar.

\section{Historia e información básica de las fincas}

A excepción de una familia, la totalidad de ellas han residido en la cuenca del río Piedras durante varias generaciones. Sin embargo, la mayoría de estas fincas fueron adquiridas por los actuales propietarios durante los últimos 20 años. Las parejas de los propietarios son a menudo inmigrantes de otras regiones de Colombia, la mayoría de ellos provenientes del Departamento del valle del Cauca, donde se ubica la cuenca del río Piedras. Siete de las diez fincas estudiadas son miembros de ASOCAMPO desde su fundación en 2001. La finca El Paraíso, se unió en 2003 y la finca Peña posteriormente en 2010. Al momento de esta investigación, un total de 64 fincas componen el universo de asociados.

Las fincas seleccionadas para el estudio se distribuyen en toda la cuenca del río Piedras. En la parte más baja de esta, se ubica la finca Cajamarca, a 1.700 metros sobre el nivel del mar, mientras que en la parte más alta, se localiza la finca Santa Marta, a 2.750 metros de altitud. Las otras fincas en estudio se sitúan entre los 2.200 a 2.500 metros de altitud. EI 
Preguntas utilizadas para conocer el estado de la finca, la situación actual y las expectativas futuras

\begin{tabular}{|c|c|}
\hline 1. & Contexto (Génesis) \\
\hline 1.1 & ¿Desde cuándo existe la Finca? \\
\hline 1.2 & ¿Cuál es el origen de la familia? \\
\hline 1.3 & ¿Desde cuándo es la finca miembro de ASOCAMPO? \\
\hline 2. & Situación Actual \\
\hline 2.1 & Altitud sobre el mar en que se encuentra la finca(m) \\
\hline 2.2 & Tamaño (ha) \\
\hline 2.3 & $\begin{array}{l}\text { Porcentaje de la finca según uso: uso agrícola, ganadero, superficie de protección (en } \\
\text { porcentaje o hectáreas) }\end{array}$ \\
\hline 2.4 & Número de vacas, ovejas, caballos, gallinas, conejos, cuyes existentes en la finca \\
\hline 2.5 & Uso del ganado vacuno (leche/carne) y producción de leche (litro/día y año) \\
\hline 2.6 & ¿Que estrategias son utilizadas para la comercialización de los productos? \\
\hline 2.7 & ¿Cómo se distribuye el trabajo en la finca? (Hombre/Mujer, Ingresos diarios) \\
\hline 2.8 & $\begin{array}{l}\text { ¿Qué porcentaje de la producción se destina al consumo personal y qué porcentaje es } \\
\text { vendido? }\end{array}$ \\
\hline 2.9 & $\begin{array}{l}\text { ¿Existen otras fuentes de ingreso en la finca aparte del proveniente de la actividad agrí- } \\
\text { cola? }\end{array}$ \\
\hline 2.10 & $\begin{array}{l}0 \text { Infraestructura existente en el hogar (sanitario, electricidad, teléfono, televisor, internet, } \\
\text { telefonía móvil) }\end{array}$ \\
\hline 2.11 & 1 Cartografía de la finca \\
\hline 2.12 & 2 Cartografía de la casa \\
\hline 2.13 & $\begin{array}{l}3 \text { Radio de acción de la familia (cuan seguido viajan a Popayán, Bogotá, viaje más largo } \\
\text { hasta el momento) }\end{array}$ \\
\hline 2.14 & 4 ¿Cuál es el mayor riesgo que consideran para el futuro? \\
\hline 3. $\mathrm{S}$ & Situación futura \\
\hline 3.1 & ¿Qué cambios ha evidenciado en los últimos años o décadas? \\
\hline 3.2 & ¿Cuándo o desde cuándo ha observado estos cambios? \\
\hline 3.3 & $\begin{array}{l}\text { De acuerdo a las respuestas de la pregunta } 3.1 \text { detalle los cambios percibidos en el cli- } \\
\text { ma y en el contexto socioeconómico. }\end{array}$ \\
\hline 3.4 & ¿Qué ha cambiado para usted desde que pertenece a ASOCAMPO? \\
\hline 3.5 & $\begin{array}{l}\text { ¿Qué medios son usados para informarse en la finca? (Libros, Periodicos, Televisión, In- } \\
\text { ternet) }\end{array}$ \\
\hline 3.6 & $\begin{array}{l}\text { ¿Qué estrategias de adaptación existen actualmente en la finca? ¿Cuáles se planean para } \\
\text { el futuro? }\end{array}$ \\
\hline 3.7 & ¿Cuáles son las expectativas para el futuro en la finca? \\
\hline 3.8 & Si usted ganara un premio de 10 millones de pesos, ¿que haría con ese dinero? \\
\hline
\end{tabular}

Fuente: Elaboración propia.

área ocupada por cada finca promedia 20 ha. Mientras la familia Escobar, propietaria de la finca Santa Marta tiene una propiedad de 35 hectáreas, la familia Conejo debe sobrevivir con menos de 2 ha. En general, el tamaño de las fincas aumenta con la altitud. Entre un $10 \%$ a $20 \%$ de la superficie total es destinada a la protección del bosque, en el marco 
Cuadro $\mathrm{N}^{\circ} 2$

Datos básicos de las fincas

\begin{tabular}{|c|c|c|c|c|c|c|c|c|c|c|}
\hline & $\mathrm{Cm}$ & FDC & EJ & EL & EM & EPal & EPar & LC & $\mathrm{P}$ & SM \\
\hline Altitud (m) & 1.700 & 2.500 & 2.400 & 2.207 & 2.490 & 2.380 & 2.300 & 2.460 & 2.500 & 2.720 \\
\hline Superficie (ha) & 4,9 & $1,5-2$ & 7,5 & 2 & 14 & 11 & 5 & 10 & 28 & 35 \\
\hline \multicolumn{11}{|c|}{ Porcentaje aproximado según tipos de uso } \\
\hline Agricultura & s.i. & s.i. & 3 & 15 & 7 & 5 & 10 & 17 & 0 & 15 \\
\hline Ganadería & s.i. & s.i. & 83 & 75 & 71 & 77 & 70 & 60 & 29 & 70 \\
\hline $\begin{array}{l}\text { Superficie de } \\
\text { protección }\end{array}$ & s.i. & s.i. & 14 & 10 & 21 & 18 & 20 & 17 & 71 & 15 \\
\hline \multicolumn{11}{|c|}{ Número de Animales } \\
\hline Vacas & 7 & 4 & 3 & 3 & 6 & 11 & 3 & 7 & 7 & 8 \\
\hline Terneros & 5 & 1 & 2 & 3 & 7 & - & 3 & 4 & 12 & . \\
\hline Ovejas & - & - & - & - & - & - & 1 & 10 & 3 & 2 \\
\hline Caballos & - & 3 & - & - & 1 & - & 2 & 3 & 2 & 3 \\
\hline Gallinas & 15 & 22 & 14 & 25 & 21 & 17 & 80 & 20 & 15 & 25 \\
\hline Conejos & - & - & - & 5 & 4 & - & - & 9 & - & 10 \\
\hline Cuyes & - & - & - & 12 & - & - & - & 6 & - & - \\
\hline Truchas & - & - & - & 150 & 60 & 700 & - & - & - & 1800 \\
\hline \multicolumn{11}{|c|}{ Producción lechera } \\
\hline $\begin{array}{l}\text { Producción de } \\
\text { leche por vaca } \\
\text { (litros por día) }\end{array}$ & 8 & $3-4(8)$ & $6-10$ & -8 & $5-7$ & $5-6$ & 10 & $5-8$ & $4-5$ & 5 \\
\hline
\end{tabular}

Fuente: Elaboración propia.

de los objetivos de la Red de Reservas. En el caso de la finca Peña se produce una excepción; $70 \%$ de su superficie se encuentra protegida. El Cuadro $N^{\circ} 2$ resume la información básica de las fincas estudiadas.

\section{Estrategias para la agricultura}

Al observar el Cuadro $\mathrm{N}^{\circ} 2$ queda en evidencia, que todas las fincas estudiadas se orientan especialmente a la ganadería, relegando el desarrollo de cultivos a un segundo lugar. Lo anterior, se desprende tanto del área disponible para cada tipo de uso y de la importancia económica que estas actividades representan. Más de un $80 \%$ de la superficie de cada finca (excluidas las áreas protegidas) son usadas exclusivamente para pastos y praderas. Algunas de estas áreas corresponden a un uso mixto de pastos y árboles (silvo-pastoril). El ganado representa el recurso principal de las familias, cada una posee entre 3 a 11 vacas. Estas son usadas principalmente para la producción de leche; dado que la producción de carne no es relevante. Cada vaca genera entre 3 a 10 litros de leche por día, parte de la cual es procesada y convertida en queso. La leche y el queso son la principal fuente de ingresos de las familias. La mayoría de ellas venden los productos directamente en el mercado de Popayán (algunos una o dos veces por semana, algunas familias incluso más seguido). Solo una finca, El Palmichal utiliza un intermediario para comercializar sus productos. 
Por otro lado, en todas las fincas estudiadas, la presencia de aves de corral es un elemento importante tanto para el consumo familiar como para la comercialización de huevos, en algunos casos. Algunas familias poseen también caballos, ovejas, conejos o cuyes. El cultivo de truchas es también una actividad popular, la mayor parte de la producción es destinada al autoconsumo, solo en caso de existir excedentes estos son vendidos en el mercado.

\section{Modo de vida, infraestructura y medios de comunicación}

Siete de las diez fincas analizadas están conectadas a la red pública de electricidad; dos familias usan paneles solares para abastecerse de energía (Cuadro $N^{\circ} 2$ ). Una de las familias vive todavía sin electricidad, siendo también la única sin suministro funcional de agua. Al momento de la investigación, ocho de las fincas poseían infraestructura sanitaria moderna y adecuada (baño completo y ducha).

Todas las familias tienen acceso a radio con batería, lo que les permite cierta autonomía en su uso. Por su parte, debido a que el acceso a la electricidad se encuentra en franco aumento, la televisión ha comenzado a tomar importancia para las familias, 7 de ellas poseen una en casa. En cuanto al uso de medios escritos de información, solo 4 familias señalaron comprar periódicos durante los viajes al mercado de Popayán para informarse. Otros medios de información como Internet tienen un rol menor, aunque en aumento, sobre todo entre los niños que la utilizan en la escuela o durante sus estadías en Popayán. Otras fuentes de información mencionadas fueron la propia ASOCAMPO, libros, conversaciones con amigos y el teléfono celular (existente en todas las fincas).

El trabajo en la finca se divide tradicionalmente de acuerdo al género; las mujeres son responsables del mantenimiento de la casa, mientras que el hombre se encarga del trabajo pesado. En algunos casos el trabajo en la finca es compartido. Los roles de cada integrante de la familia dependen de las necesidades de mano de obra, las necesidades específicas del hogar y si el miembro de la familia tiene otro trabajo. La mitad de las fa- milias entrevistadas perciben ingresos extras (pensiones, préstamos de familiares, trabajo en otras fincas o trabajos en la construcción de la carretera). Por otro lado, en cuanto a la movilidad, el radio de acción de los entrevistados varía considerablemente; mientras cuatro familias (o al menos un miembro de ellas) han estado alguna vez en la capital Bogotá, una en Ecuador y una en la costa Caribe, algunos solo han viajado a los alrededores de cuenca del río Piedras.

\section{Cambios percibidos en las últimas décadas}

Siete de las diez familias señalaron haber evidenciado un aumento en las precipitaciones estivales durante los años previos. Asimismo, cinco de estas mismas señalaron que es difícil establecer las estaciones del año, debido al carácter homogéneo que estas han desarrollado. Por otro lado, dos familias señalaron reconocer un aumento generalizado de las precipitaciones y también un mayor número de eventos de precipitaciones extremas, así como fuertes vientos y el aumento de periodos de sequía. Todos estos fenómenos climáticos son vistos como la causa de cambios en los modos de vida y en la economía, aunque los entrevistados no fueron capaces de definir la dimensión temporal en que estos cambios han acontecido.

Como efectos negativos de las condiciones de producción se señaló el aumento de plagas y dificultades para determinar los momentos correctos de siembra y cosecha. Como efectos positivos, la posibilidad de cultivar productos termófilos a una mayor altitud, como café, banano, mango y aguacate. Este puede ser considerado como un indicador de aumento de la temperatura, el cual no es percibido directamente. Solo una de las familias percibió más granizadas, bajas temperaturas y menos precipitaciones, así como un aumento de la radiación solar y un aumento de la erosión.

En cuanto a cambios socioeconómicos, la solución del conflicto entre campesinos e indígenas fue señalado como el cambio más importante. Por otro lado, algunos campesinos se ven afectados por los bajos precios de comercialización de sus productos, debido al 
aumento de la competencia causada por la importación de productos más baratos desde Ecuador. Una de las familias señaló que la construcción de la carretera a Popayán ha contribuido a mejorar la calidad de vida de las personas. Otros cambios identificados son el empeoramiento de la salud de los animales y la desmotivación de la población.

Los cambios en las técnicas de agricultura y las estrategias de marketing introducidas por ASOCAMPO son vistas como efectos positivos por la gran mayoría. Solo una persona señaló no identificar ningún efecto positivo tras la incorporación a ASOCAMPO. El fortalecimiento de las capacidades técnicas fue nombrado en la mayoría de los casos, lo cual se relaciona con la transformación a la economía orgánica. La solución del conflicto anteriormente mencionado, es también atribuida a ASOCAMPO.

La conservación del bosque fue mencionada por dos familias, como también las ayudas financieras y el mejor marketing $y$ visibilidad impulsada por ASOCAMPO. Otros puntos mencionados fue la promoción del ecoturismo, mejoramiento de la infraestructura y de la calidad de vida y un mayor sentido de comunidad.

\section{Adaptaciones a los cambios percibidos}

Las estrategias de adaptación nombradas por los entrevistados se tratan mayoritariamente de adaptación al cambio climático y la mayoría de ellas se relacionan con la membrecía a ASOCAMPO. La agricultura orgánica y la diversificación de la gama de productos pueden ser consideradas como una adaptación al cambio climático y también al cambio socioeconómico (aumento de la calidad y mayor diversidad de productos para competir con las exportaciones baratas). La implementación de cercos vivos y techos plásticos contra la lluvia y el granizo son buenos ejemplos de toma de conciencia de la población ante estos nuevos desafíos y son una medida que ha permitido aumentar la productividad $y$ preservar el paisaje cultural en general, independiente del cambio climático. Además, las experiencias de los campesinos indican la reducción de los problemas con plagas. Finalmente, solo una persona entrevistada señaló no aplicar ninguna medida de adaptación.

\section{Prioridades para el desarrollo futuro}

Ante la situación ficticia de tener mucho dinero, la mayoría de las familias entrevistadas, opinó que si ganaran un monto de 10 millones de pesos colombianos (aprox. US \$5.700), lo invertirían en la finca o comprarían más tierras o animales, otra opción considerada es mejorar las técnicas de producción y comprar maquinarias. La compra de nuevo mobiliario para el hogar e incluso la reconstrucción de la casa, fueron también mencionadas. Tres de los entrevistados viajarían en Colombia o a Europa y dos invertirían en ecoturismo, construyendo un hotel o un camping. Solo dos entrevistados compartirían el dinero con otras personas (hijos, vecinos).

En cuanto al futuro de la finca, tres familias piensan heredarla en partes iguales entre todos sus hijos, solo una la cederá a uno de todos sus posibles herederos. Lo anterior se debe a una tendencia creciente de migración de los hijos a la ciudad, en muchos casos para realizar estudios en la universidad, lo cual es una fuerza expulsora de población que actualmente se presenta con mayor recurrencia en la zona.

\section{Conclusiones}

Esta investigación ha demostrado que el reforzamiento del capital social puede ser un estímulo importante para asegurar la paz y el desarrollo sustentable en una región rural. A pesar de que el capital financiero y material de los campesinos del río Piedras es limitado, estos optimizan el uso de sus recursos naturales y humanos, considerando la conservación de los servicios ecosistémicos que tanto ellos como otras personas utilizan, permitiendo una mejor preparación ante los cambios producidos, tanto por el cambio climático, como por el socioeconómico. Retomando los objetivos mencionados al principio de este trabajo, los principales resultados obtenidos son los siguientes:

Los campesinos han evidenciado actualmente cambios en las condiciones climáticas en comparación a las décadas precedentes. La evidencia más importante es el cambio de la temporada lluviosa con la seca, lo que produce un problema para identificar las estaciones, 
establecer los periodos de cosecha y siembra, lo cual ha provocado también un aumento de las plagas. Desde la perspectiva positiva, el aumento de temperatura ha permitido el cultivo de plantas termófilas en áreas más altas, como por ejemplo el plátano y el café.

El aumento del capital social (Coleman, 1988) y los programas de apoyo de la Fundación Río Piedras en la zona han permitido la introducción de técnicas de agricultura ecológica sustentable, como la rotación de pastos, la producción y uso de compost, la instalación de barreras contra el viento y la construcción de terrazas. Asimismo, ha permitido el desarrollo de casas sustentables. De igual forma el trabajo en comunidad, como por ejemplo en la construcción de barreras antierosión en la carretera, ha mejorado la comunicación entre vecinos e influenciado positivamente el espíritu de comunidad. De acuerdo a la percepción de los campesinos, la introducción de estas medidas y el refuerzo del capital social han mejorado considerablemente las condiciones de vida. Sin embargo, resulta difícil cuantificar esta mejora en términos de ingresos $u$ otros indicadores.

Las estrategias implementadas son consideradas como una adaptación al cambio climático, lo cual se corrobora con la existencia de cultivos de plátano o café en esta altitud. No obstante, 10 años es un periodo muy corto para establecer con precisión cambios en el clima. Un periodo de observación más extenso en combinación con el análisis de datos climáticos, son necesarios por verificar esta situación descrita por los campesinos.

Considerando estos antecedentes es posible señalar que el modelo desarrollado en la cuenca del río Piedras puede ser adaptado para su aplicación en otras regiones de montaña colombiana con conflictos similares. Las zonas de amortiguación o de desarrollo en reservas de la biósfera son lugares ideales para este tipo de iniciativas, las cuales no debieran limitarse solo a estos espacios, sino que debieran ser adaptadas y aplicadas en otros contextos locales.

\section{Referencias bibliográficas}

AUSTRIAN MAB COMMITTEE (editors). Biosphere Reserves in the Mountains of the
World. Excellence in the clouds? Vienna: MAB, 2011.

BATISSE, M. The Biosphere Reserve: A Tool for Environmental Conservation and Management. Environmental Conservation, 1982, Vol. 2, Nº 9, p. 101-111.

BATISSE, M. Developing and Focusing the Biosphere Reserve Concept. Nature and Resources, 1986, Vol. 3, N²2, p. 2-11.

BATISSE, M. Biosphere Reserves: A Challenge for Biodiversity Conservation and Regional Development. Environment: Science and Policy for Sustainable Development, 1997, Vol. 5, No 39, p. 6-33.

BORSDORF, A.; BORSDORF, F. \& ORTEGA, L.A. Towards climate change adaptation, sustainable develop-ment and conflict resolution - the Cinturón Andino Biosphere Reserve in Southern Colombia. eco.mont-Journal on Protected Mountain Areas Research and Management, 2011, Vol. 2, No 3, p. 43-48.

BRYANT, R. L. \& BAILEY, S. Third World Political Ecology. London: Routledge, 1997.

CAMPBELL, L. M. \& VAINIO-MATTILA, A. Participatory Development and CommunityBased Conservation: Opportunities Missed for Lessons Learned? Human Ecology, 2003, Vol. 3, $\mathrm{N}^{\circ} 31$, p. 417-437.

COLEMAN, J. S. Social Capital in the Creation of Human Capital. American Journal of Sociology, 1988, No 94, p. 95-120.

ECHAVARRÍA, M. Water user associations in the Cauca Valley: A voluntary mechanism to promote upstream-downstream cooperation in the protection of rural watersheds. Rome: FAO, 2002.

FIGUEROA CASAS, A. y VALENCIA ROJAS, M. Fragmentación y coberturas vegetales de ecosistemas andinas, departamento del Cauca. Popayán: Editorial Universidad del Cauca, 2009.

HARDEN, C. P. Human impacts on headwater fluvial systems in the northern and central Andes. Geomorphology, 2006, № 79, p. 249-263. 
INSTITUTO DE HIDROLOGÍA, METEOROLOGÍA Y ESTUDIOS AMBIENTALES (IDEAM). El Macizo Colombiano y su Área de Influencia. República de Colombia. Bogotá: Ministerio del Medio Ambiente. Instituto de Hidrología, Meteorología y Estudios Ambientales.

LANGE, S. Leben in Vielfalt. UNESCOBiosphä-renreservate als Modellregionen für ein Miteinander von Mensch und Natur. Vienna, 2005.

LANGHOLZ, J. A.; LASSOIE, J. P.; LEE, D. \& CHAPMAN, D. Economic considerations of privately owned parks. Ecological Economics, 2000, Vol. 2, No 33, p. 173-183.

LANGHOLZ, J. A. \& LASSOIE, J.P. Perils and Promise of Privately Owned Protected Areas. BioScience, 2001, Vol. 12, № 51, p. 1079-1085.

LITTLE, M. A.; BAKER, P. T. \& IVES, J. D. Planning and Development of Man and the Biosphere (MAB) Research in the Andes. Mountain Research and Development, 1981, Vol. 2, Nº1, p. 103-114.

PRICE, M. Ecopolitics and environmental nongovernmental organizations in Latin Ame- rica. The Geographical Review, 1994, Vol. 1, $\mathrm{N}^{\circ} 84$, p. $42-58$.

SALAMON, L. M.; ANHEIER, H. K.; LIST, R.; TOEPLER, S. \& WOJCIECH SOKOLOWS$\mathrm{KI}, \mathrm{S}$. Global Civil Society. Dimensions of the Nonprofit Sector. Baltimore: The John Hopkins Center for Civil Society Studies, 1999.

SCHAAF, T. Biosphere Reserves: Tangible and Intangible Values. In: HARMON, D. \& PUTNEY, A. D. (editors). The full value of parks. From economics to the intangible. Lanham: Rowman \& Littlefield, 2003, p. 185-196.

THIEL, L. \& EFFLER, D. The Sierra Nevada de Snata Marta Biosphere Reserve, Colombia, the origin of Coffee K.U.L.T. In: AUSTRIAN MAB COMMITTEE (editor). Biosphere Reserves in the Mountains of the World. Excellence in the Clouds? Vienna: Austrian Academy of Sciences Press, 2011, p. 93-95.

VILARDY, S. y GONZÁLEZ, J.A. (editores). Repensando la Ciénaga: Nuevas miradas y estrategias para la sostenibilidad en la Ciénaga Grande de Santa Marta. Santa Marta: Universidad del Magdalena y Universidad Autónoma de Madrid, 2011. 